\title{
COMMENTARY
}

\section{Revisiting the merits of nifedipine}

\author{
Motoaki Sano \\ Hypertension Research (2011) 34, 1173-1174; doi:10.1038/hr.2011.144; published online 15 September 2011
}

$\mathrm{R}^{2}$ andomized trials of blood pressure reduction have shown a reduced risk of stroke and heart failure. Among blood pressure-lowering agents, blockers of the reninangiotensin system, such as angiotensin-converting enzyme inhibitors (ACEIs) and AT1 receptor antagonists $(\mathrm{ARBs})$, are thought to have specific properties beyond blood pressure control, which reduce cardiovascular complications. However, recent clinical trials indicated that ACEIs and ARBs are not always such all-rounders. ARBs are effective for primary prevention of paroxysmal atrial fibrillation (PAF), but have little benefit on secondary prevention of PAF and persistent or permanent atrial fibrillation. ARBs are cardioprotective in patients with heart failure and reduced ejection fraction, but have no benefits on heart failure patients with preserved ejection fraction. Both patient groups have a similarly poor prognosis, but treatment options for those patients with preserved ejection fraction are limited. Moreover, very little is known about the mode of death in these patients.

Nifedipine is a long-acting L-type calcium (Ca) channel blocker and one of the most widely used medications for hypertension among such agents. ACTION (A Coronary disease Trial Investigating Outcome with Nifedipine GITS) was designed to investigate the effects of nifedipine on clinical outcomes in patients with stable symptomatic coronary disease. ${ }^{1}$ Briefly, 7665 patients aged 35 years or older with stable angina requiring treatment were randomly assigned to treatment with either nifedipine $(n=3825)$ or matching placebo $(n=3840)$ in addition to their existing medication. Nifedipine reduced the incidence of new overt heart failure by $29 \%$.

Dr M Sano is at the Department of Cardiology, Keio University School of Medicine, 35 Shinanomachi Shinjuku-ku, Tokyo 160-8582, Japan.

E-mail: msano@a8.keio.jp
Notably, there was a trend for nifedipine to be more effective than placebo in preventing heart failure patients with preserved ejection fractions $(\geqslant 45 \%)$. $^{2}$ Based on this subanalysis of ACTION trials, Diastolic Heart Failure Management by Nifedipine (DEMAND) is now ongoing in Japan to evaluate the effects of sustained-release nifedipine on the clinical composite response endpoint in patients with heart failure with preserved ejection fraction (https://upload.umin.ac.jp/cgi-open-bin/ctr/ctr. cgi? function=brows\&action $=$ brows \& type $=$ summary\&recptno $=$ R000004634\&language $=\mathrm{J}$ ).

Diastolic dysfunction is often present in elderly subjects with hypertension and diabetes, which often share conditions including obesity and left ventricular (LV) hypertrophy. These comorbidities could synergistically affect LV diastolic function. Anti-obesity action of short-term treatment with nifedipine on obese and diabetic rats has been reported. Radin et al. ${ }^{3}$ demonstrated that nifedipine improved systolic pressure and glycemic control in 9-month-old obese and hypertensive female SHHF/Mcc-fa (cp) rats, effects that are associated with a marked loss in body weight and fat mass and improved lipid metabolism. Nifedipine significantly decreased body weight in obese females compared with ACEIs enalapril. Iwai et al. ${ }^{4}$ also showed that non-hypotensive doses of nifedipine enhanced insulin sensitivity and reduced adipose tissue in 9-week-old diabetic male KK-Ay mice. However, the long-term effects of nifedipine on body weight, adipose tissue mass and insulin sensitivity in obese subjects remains unexamined.

In the current issue of Hypertension Research, Tian et al. ${ }^{5}$ reported that non-hypotensive doses of nifedipine $\left(1 \mathrm{mg} \mathrm{kg}^{-1} \mathrm{day}^{-1}\right.$ for 4 months) significantly reduced epididymal white adipose tissue and body weight gain in mild-obese endothelial nitric oxide synthase (eNOS)-deficient mice. Nifedipine- treated mice exhibited increased wholebody oxygen consumption rates (VO2), a decreased respiratory ratio (greater utilization of fat as an energy source) and an increase in the locomotor activity in dark time, relative to untreated mice, whereas food intake per weight was comparable. Plasma insulin level and HOMA-IR (homeostatic model assessment-insulin resistance) index were significantly decreased in the nifedipine-treated mice. These findings indicated that nifedipine treatment increases the ability to oxidize lipid and improves insulin sensitivity.

Obesity is associated with a decrement in the ability of skeletal muscle to oxidize lipid, which links to weight gain. A reduction in fatty acid oxidation and resulting lipid accumulation in skeletal muscle induces insulin resistance. Of note, they found that nifedipine treatment increased mRNA expression of peroxisome proliferator-activated receptor (PPAR) $-\gamma$ coactivator $1 \alpha$ (PGC- $1 \alpha$ ) in eNOS-deficient mice. PGC- $1 \alpha$ regulates nuclear respiratory factor-1 (NRF-1)-, PPARand estrogen-related receptor-dependent transcription, increases expression of both nuclear and mitochondrial-encoded genes of oxidative metabolism (for example tricarboxylic acid cycle, lipid oxidation and electron transport complexes) and induces mitochondrial biogenesis. PGC- $1 \alpha$ also upregulates the expression of glucose transporter-4 (GLUT4) via binding and coactivating muscle-specific transcription factor MEF2C and increases glucose transport rate in murine $\mathrm{C} 2 \mathrm{C} 12$ myotube. ${ }^{6}$ There is a compelling body of evidence for mitochondrial dysfunction in association with insulin resistance and obesity. Indeed, skeletal muscle-specific knockout animals for PGC- $1 \alpha$ have a significant shift from oxidative towards glycolytic muscle fibers. ${ }^{7}$ Most importantly, reduced expression of PGC-1 $\alpha$ and NRF-dependent genes in skeletal muscle is the characteristic feature of both diabetic subjects and family history-positive non-diabetic subjects. ${ }^{8}$ These 
transcriptional changes lead to the metabolic disturbances characteristic of insulin resistance and type 2 diabetes, which include decreased fat oxidation with increased lipid esterification and accumulation in skeletal muscle. It would be intriguing to know whether the stimulatory effect of nifedipine on PGC-1 expression is universal and whether nifedipine treatment could restore the oxidative capacity of mitochondria in obese and diabetic subjects. Impairing the augmentation of mitochondrial oxidative phosphorylation in the diabetic mitochondria contributes to a decreased rate of LV relaxation during increased workload. Future studies are thus necessary to examine the impact of nifedipine on mitochondrial function, as well as on the expression of PGC- $1 \alpha$ and its downstream target genes in cardiomyocytes, in terms of the restoration of diastolic dysfunction by nifedipine.

Other possible mechanisms for improving insulin sensitivity by nifedipine include the acceleration of adipocyte differentiation. Acceleration of adipocyte differentiation induces smaller adipocytes and $\operatorname{PPAR} \gamma$ is a potent inducer of adipocyte differentiation. Nifedipine increased PPAR $\gamma$ expression in white adipose tissues of type 2 diabetic $\mathrm{KK}-\mathrm{A}^{\mathrm{y}}$ mice, as well as adiponectin, insulin receptor, insulin receptor substrate-1 and GLUT-4 expression. ${ }^{4}$ Activity of NADPH oxidase decreased, whereas activity of superoxide dismutase increased in white adipose tissues for nifedipine-administered $\mathrm{KK}-\mathrm{A}^{\mathrm{y}}$ mice. ${ }^{4}$ Furthermore, some dihydropyridine calcium channel blockers, including nifedipine, have mineralocorticoid receptor (MR) antagonist activity. ${ }^{9,10}$ Both PPAR $\gamma$ activation and MR blockage could shift macrophage polarity from proinflammatory (M1) to an alternatively activated form. ${ }^{11}$ Adipose tissue macrophages are both necessary and sufficient for the development of insulin resistance with obesity. Thus, a unique beneficial aspect of nifedipine with respect to insulin sensitivity could be mediated, at least in part, through the modulation of macrophage polarity.

\section{CONFLICT OF INTEREST}

The author declares no conflict of interest.

1 Poole-Wilson PA, Lubsen J, Kirwan BA, van Dalen FJ, Wagener G, Danchin N, Just H, Fox KA, Pocock SJ, Clayton TC, Motro M, Parker JD, Bourassa MG, Dart AM, Hildebrandt P, Hjalmarson A, Kragten JA, Molhoek GP, Otterstad JE, Seabra-Gomes R, SolerSoler J, Weber S. Effect of long-acting nifedipine on mortality and cardiovascular morbidity in patients with stable angina requiring treatment (action trial): randomised controlled trial. Lancet 2004; 364: 849-857.

2 Sutton GC, Erik Otterstad J, Kirwan BA, Voko Z, de Brouwer S, Lubsen J, Poole-Wilson PA. The development of heart failure in patients with stable angina pectoris. Eur J Heart Fail 2007; 9: 234-242.

3 Radin MJ, Chu YY, Hoepf TM, McCune SA. Treatment of obese female and male shhf/mcc-fa(cp) rats with antihypertensive drugs, nifedipine and enalapril: effects on body weight, fat distribution, insulin resistance and systolic pressure. Obes Res 1993; 1: 433-442.
4 Iwai M, Kanno H, Inaba S, Senba I, Sone H, Nakaoka $\mathrm{H}$, Horiuchi M. Nifedipine, a calcium-channel blocker, attenuated glucose intolerance and white adipose tissue dysfunction in type 2 diabetic kk-a(y) mice. Am J Hypertens 2011; 24: 169-174.

5 Tian Z, Miyata K, Tabata M, Yano M, Tazume H, Aoi J, Takahashi O, Araki K, Kawasuji M, Oike Y. Nifedipine increases energy expenditure by increasing PGC- $1 \alpha$ expression in skeletal muscle. Hypertens Res 2011; 34: 1221-1227.

6 Michael LF, Wu Z, Cheatham RB, Puigserver $P$, Adelmant G, Lehman JJ, Kelly DP, Spiegelman BM. Restoration of insulin-sensitive glucose transporter (glut4) gene expression in muscle cells by the transcriptional coactivator pgc-1. Proc Natl Acad Sci USA 2001; 98: 3820-3825.

7 Handschin C, Chin S, Li P, Liu F, Maratos-Flier E, Lebrasseur NK, Yan Z, Spiegelman BM. Skeletal muscle fiber-type switching, exercise intolerance, and myopathy in pgc-1alpha muscle-specific knock-out animals. J Biol Chem 2007; 282: 30014-30021.

8 Patti ME, Butte AJ, Crunkhorn S, Cusi K, Berria R, Kashyap S, Miyazaki Y, Kohane I, Costello M, Saccone R, Landaker EJ, Goldfine AB, Mun E, DeFronzo R, Finlayson J, Kahn CR, Mandarino LJ. Coordinated reduction of genes of oxidative metabolism in humans with insulin resistance and diabetes: potential role of pgc1 and nrf1. Proc Natl Acad Sci USA 2003; 100: 8466-8471.

9 Dietz JD, Du S, Bolten CW, Payne MA, Xia C, Blinn JR, Funder JW, Hu X. A number of marketed dihydropyridine calcium channel blockers have mineralocorticoid receptor antagonist activity. Hypertension 2008; 51: 742-748.

10 Matsui T, Takeuchi M, Yamagishi S. Nifedipine, a calcium channel blocker, inhibits inflammatory and fibrogenic gene expressions in advanced glycation end product (age)-exposed fibroblasts via mineralocorticoid receptor antagonistic activity. Biochem Biophys Res Commun 2010; 396: 566-570.

11 Usher MG, Duan SZ, Ivaschenko CY, Frieler RA, Berger S, Schutz G, Lumeng CN, Mortensen RM. Myeloid mineralocorticoid receptor controls macrophage polarization and cardiovascular hypertrophy and remodeling in mice. J Clin Invest 2010; 120: 3350-3364. 